\title{
Application of computed tomography-based radiomics signature analysis in the prediction of the response of small cell lung cancer patients to first-line chemotherapy
}

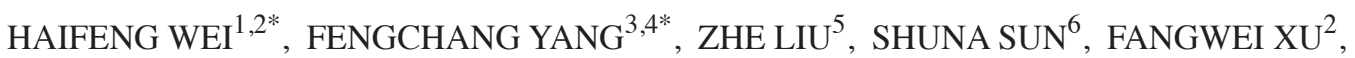

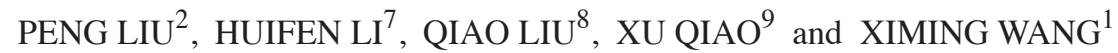

\begin{abstract}
${ }^{1}$ Diagnostic Room of Computer Tomography, Shandong Medical Imaging Research Institute, Shandong Provincial Key Laboratory of Diagnosis and Treatment of Cardio-Cerebral Vascular Disease, Shandong University, Jinan, Shandong 250021; ${ }^{2}$ Department of Radiology, Affiliated Hospital of Shandong University of Traditional Chinese Medicine, Jinan, Shandong 250012; ${ }^{3}$ Department of Radiology, Shandong Cancer Hospital Affiliated to Shandong University; ${ }^{4}$ Department of Radiology, Shandong Academy of

Medical Sciences, Jinan, Shandong 250117; Departments of ${ }^{5}$ Pharmacy and ${ }^{6}$ Dermatology, Affiliated Hospital of

Shandong University of Traditional Chinese Medicine, Jinan, Shandong 250012; ${ }^{7}$ Department of Natural Drugs,

School of Pharmacy, Shandong University of Traditional Chinese Medicine, Jinan, Shandong 250355;

${ }^{8}$ Key Laboratory of Experimental Teratology, Ministry of Education and Department of Molecular

Medicine and Genetics, Shandong University School of Medicine, Jinan, Shandong 250012; ${ }^{9}$ Department

of Biomedical Engineering, Shandong University, Jinan, Shandong 250061, P.R. China
\end{abstract}

Received April 12,2018; Accepted January 16, 2019

DOI: $10.3892 /$ etm.2019.7357

\begin{abstract}
The aim of the present study was to investigate the utility of a computed tomography (CT)-based radiomics signature for the early prediction of the tumor response of small cell lung cancer (SCLC) patients to chemotherapy. A dataset including 92 patients from a clinical trial was retrospectively assembled. All of the patients received the standard first-line regimen of etoposide and cisplatin. According to the Response Evaluation Criteria in Solid Tumors 1.1, the patients were divided into two groups: Response and no response groups. A total of 21 radiomics features were extracted from $\mathrm{CT}$ images prior to and after two cycles of chemotherapy and a radiomics signature was constructed via a binary logistic
\end{abstract}

Correspondence to: Professor Ximing Wang, Diagnostic Room of Computer Tomography, Shandong Medical Imaging Research Institute, Shandong Provincial Key Laboratory of Diagnosis and Treatment of Cardio-Cerebral Vascular Disease, Shandong University, Radiology Building, 324 Jingwu Road, Jinan, Shandong 250021, P.R. China

E-mail: wangximing1969@163.com

Dr Xu Qiao, Department of Biomedical Engineering, Shandong University, 17923 Jingshi Road, Jinan, Shandong 250061, P.R. China E-mail: qiaoxu@sdu.edu.cn

*Contributed equally

Key words: radiomics signature, small cell lung cancer, predictor, chemotherapy, computed tomography regression model. The area under the receiver operating characteristics curve (AUC) was determined to evaluate the performance of the radiomics signature to predict the response to chemotherapy. The clinicopathological factors associated with chemotherapy in patients with SCLC were also evaluated, and a predictive model was established using a binary logistic regression analysis. The 21 radiological features were used to establish a radiomics signature that was significantly associated with the efficacy of SCLC chemotherapy $(\mathrm{P}<0.05)$. The performance of the radiomics signature to predict the chemotherapy efficacy (AUC=0.797) was better than that of the model using clinicopathological parameters $(\mathrm{AUC}=0.670$ ). Therefore, the present study demonstrated that radiomics features may be promising prognostic imaging biomarkers to predict the response of SCLC patients to chemotherapy and may thus be utilized to guide appropriate treatment planning.

\section{Introduction}

Lung cancer is the most common type of malignant tumor and the leading cause of cancer-associated mortality worldwide $(1,2)$. Small cell lung cancer (SCLC) accounts for 15-20\% of all lung cancer cases and is characterized by rapid growth and early metastatic spread (3). Of all newly diagnosed patients with SCLC, $\sim 70 \%$ present with advanced disease and require systemic chemotherapy. In such cases, clinicians must promptly initiate treatment. However, although SCLC is sensitive to chemotherapy, with initial response rates of $\geq 60 \%$, the 5-year overall survival rate is $<5 \%$ (4). In the last decades, SCLC therapy and prognosis have not significantly improved and no novel drugs have been approved in the recent years (5), although progress has been made in the characterization of the genetic 
landscape of SCLC (6-8). In addition to continued development of novel treatments, the determination of the optimal use of the existing chemotherapies to improve the survival rate of SCLC patients represents a major clinical challenge.

Several combinations of chemotherapeutics may be used to treat SCLC. However, the etoposide-cisplatin (EP) regimen remains the primary choice of treatment and no novel chemotherapeutic combinations have been identified to be superior to EP as the first-line therapy in SCLC patients $(9,10)$. However, certain cases of SCLC do not respond well to EP chemotherapy. Thus, the possibility to predict treatment outcomes for SCLC patients, particularly those at high risk of responding poorly to first-line chemotherapy, is of great interest. This may allow for pre-chemotherapy risk stratification in SCLC and enable clinicians to select a treatment tailored to each patient's individual risk profile.

To date, no biomarkers with the ability to indicate the clinical response of SCLC patients to treatment have been identified; $75 \%$ of patients with SCLC have $\geq 2$ circulating tumor cells (CTCs) $/ 7.5 \mathrm{ml}$ peripheral venous blood. Thus, CTC detection may be used to determine the response to therapy $(11,12)$. However, the low CTC number in blood may affect the reproducibility of these tumor cell counts. Furthermore, the currently available serum tumor markers for lung cancer cannot be used to monitor SCLC, as they have relatively low sensitivity and specificity for cancer cells; these include neuron-specific enolase (NSE), New York esophageal squamous cell carcinoma 1 antibody, plasma fibrinogen, D-dimer, carcinoembryonic antigen (CEA) and progastrin-releasing peptide (ProGRP) (13-15). The identification of novel, cost-effective and accurate biomarkers is crucial for predicting the clinical response of SCLC patients to chemotherapy.

Previous radiological studies have performed large-scale data analyses to improve the utilization of imaging over the past decade. High-throughput medical image analysis has been performed for quantitative feature extraction. From the images, certain features are being extracted and converted into data, which may in turn be analyzed using a decision support system; this novel technology is known as radiomics (16). This method is particularly useful in solid tumors that are unevenly shaped. Radiomics is able to capture heterogeneity in a non-invasive and cost-effective way (17-19). In fact, it is more useful than biopsy in this regard, as it reveals heterogeneity across the entire tumor (20).

Computed tomography (CT) is the most commonly used imaging modality, it is able to quantify tissue density with a high resolution and provide clear tumor images through enhanced scanning (19). In recent years, radiomics research has greatly advanced with regard to CT image analysis. Previous studies have identified features associated with tumor histology (21-23), tumor staging (24), overall survival $(25,26)$ and gene mutations (27). However, to the best of our knowledge, no previous study has elucidated the prognostic value of radiomics in SCLC patients scheduled for first-line chemotherapy.

The aim of the present study was to identify a CT-based radiomics signature and investigate whether it is able to predict the clinical response of SCLC patients to first-line chemotherapy.

\section{Materials and methods}

Patient selection. A total of 134 patients with SCLC histopathologically confirmed by fine-needle aspiration biopsy and received chemotherapy at Shandong Cancer Hospital Affiliated to Shandong University (Jinan, China) were recruited for the present study. The patient inclusion criteria were as follows: i) Chemotherapy between March 2015 and March 2017; ii) No treatment or operation prior to admission; and iii) two courses of standard EP chemotherapy. Subjects with a mixed SCLC pathology $(n=3)$, as well as those who did not receive two courses of standard EP chemotherapy $(n=5)$, no availability of double-enhanced CT images captured prior to and after chemotherapy $(n=13)$ and those who were lost to follow-up ( $\mathrm{n}=21)$ were excluded (Fig. 1).

Therapeutic process and grouping standard. A total of 92 patients with SCLC who received two cycles of EP chemotherapy were included in the present study (EP regimen: cisplatin at an accumulated dose of $80 \mathrm{mg} / \mathrm{m}^{2} \mathrm{IV}$ for 3 days and $100 \mathrm{mg} / \mathrm{m}^{2} \mathrm{IV}$ etoposide daily (days 1-3). The interval between the two cycles of EP chemotherapy was 18 days. A chest CT scan was performed during the week prior to treatment initiation and at 4 weeks following routine chemotherapy. In accordance with the New Response Evaluation Criteria in Solid Tumors (RECIST) 1.1 (28), two experienced radiologists compared the CT imaging characteristics from all scans obtained prior to chemotherapy compared with the post-chemotherapy scans. Each radiologist evaluated the images in the same manner and any discrepancies were resolved by discussion until a consensus was reached. The patients were divided into the following groups: Response group $(n=70)$ and no response $(n=22)$ group. Those patients who achieved either a complete or a partial response were assigned to the response group, whereas those with stable disease or disease progression were assigned to the no response group (29).

Clinicopathological factors. The potential clinicopathological factors associated with SCLC including age $(<60$ years or $\geq 60$ years), sex, tumor extent (limited or diffuse) (30), tumor (T)-stage, nodal (N)-stage, metastasis (M)-stage, tumor location (central or peripheral), smoking (yes or no), smoking period $(\leq 10$, $11-20,21-30$, or $\geq 30$ years), smoking index $(<400$ or $\geq 400)$ and tumor laboratory indicators [ProGRP, $<54.8$ or $\geq 54.8 \mathrm{ng} / \mathrm{ml}$; $\mathrm{NSE},<3.4$ or $\geq 3.4 \mathrm{ng} / \mathrm{ml}$; CEA,$<17$ or $\geq 17 \mathrm{ng} / \mathrm{ml}$; and cytokeratin 19 fragment (Cyfra21-1), $<3.3$ or $\geq 3.3 \mathrm{ng} / \mathrm{ml}$ ] were analyzed.

CT image acquisition and pre-processing imaging environment. All patients underwent pulmonary CT examination using a Philips Brilliance 128i CT scanner (Philips Healthcare, Amsterdam, Netherlands). The tube voltage and current were $120 \mathrm{kV}$ and $220 \mathrm{~mA}$, respectively; the pitch was 1.0 and the collimator measured $64 \times 0.625 \mathrm{~mm}$. The acquired data were reconstructed into slices of $1.0 \mathrm{~mm}$ thickness at $1.0-\mathrm{mm}$ intervals. The field of view was $20 \times 20 \mathrm{~cm}$. Prior to each scan, $1.5 \mathrm{ml} / \mathrm{kg}$ non-ionic contrast medium (Ultravist $300 \mathrm{mg} \mathrm{I} / \mathrm{ml}$; Schering Healthcare, Guangzhou, Guangdong, China) was injected into the antecubital vein using a $20 \mathrm{G}$ needle at a flow rate of $3 \mathrm{ml} / \mathrm{sec}$. Saline (30-40 ml) was injected at the same flow rate. CT scanning was automatically triggered using a bolus-tracking 


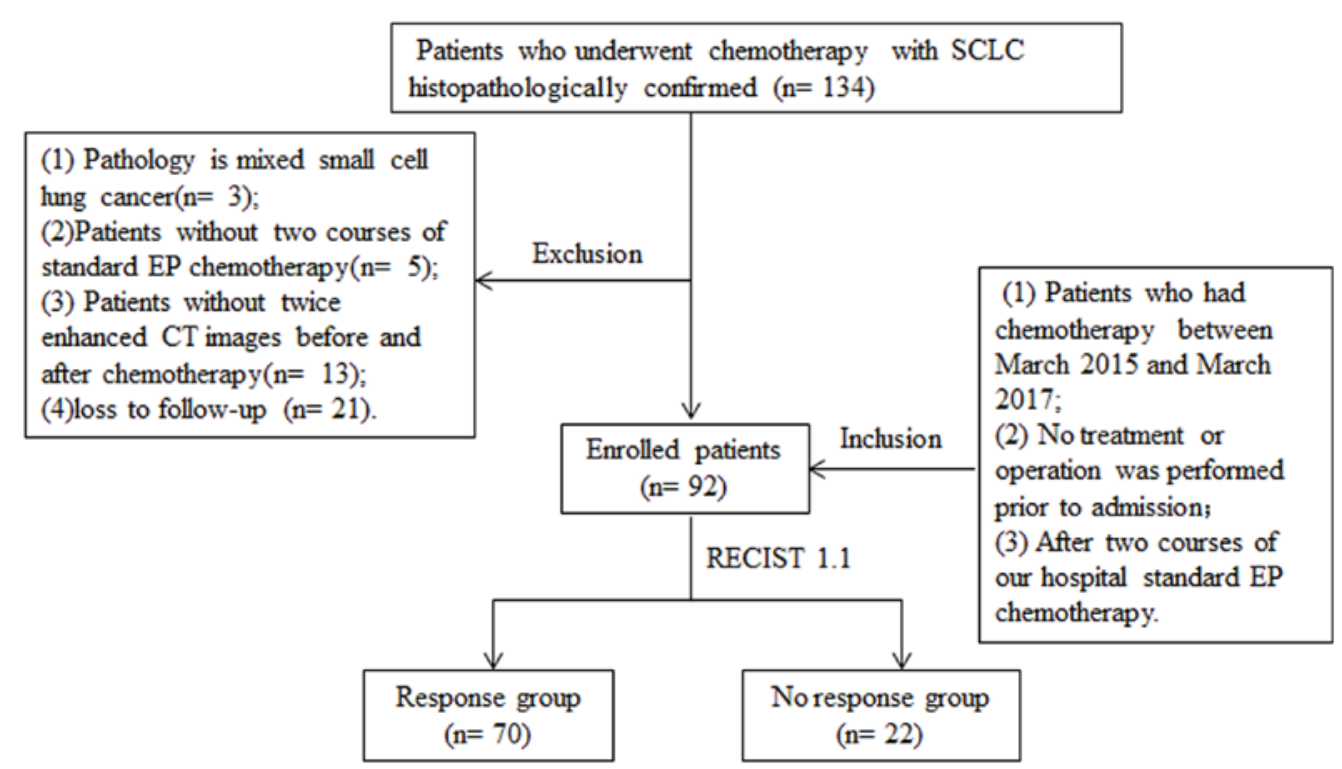

Figure 1. Flowchart of the case identification process. SCLC, small cell lung cancer; CT, computed tomography; EP, etoposide and cisplatin; RECIST, Response Evaluation Criteria in Solid Tumors.

technique following administration of the contrast agent. The region of interest was the pulmonary artery trunk and a threshold of 100 Hounsfield units was set. Scanning was triggered with a delay of $2 \mathrm{sec}$ once the threshold was reached.

Pre-processing. All original images captured prior to chemotherapy were in Digital Imaging and Communications in Medicine format (the international standard to transmit, store, retrieve, print, process and display medical imaging information). Prior to extraction of the quantitative radiomics features, the images required 3-dimensional (3D) manual segmentation. Itk-snap software (version 3.4; www.itksnap.org) was used for this purpose. First, the abdominal window was contoured to identify its boundaries with the chest wall and other soft tissues. The pulmonary window was then contoured to capture the maximum extent of the tumor in the lung parenchyma (25). All tumors were manually segmented and completed independently by two radiologists with 11 and 20 years of experience in CT imaging of thoracic malignancies, respectively. Each radiologist reviewed the segmented images and any discrepancies were resolved by discussion until a consensus was reached. All images were normalized to gray values.

Radiomic feature extraction. In SCLC, radiomic feature extraction is performed in 3D volumes of interest (VOIs) in order to calculate a set of statistical measures. These are then analyzed to determine which are statistically significant. The 3D VOIs were comprised of 3D regions of primary SCLC tumors. In each case, the 3D VOI was segmented manually by two experienced ( $\geq 10$ years experience) radiologists. The radiologists delineated the boundaries of the primary tumors on a transversal plane using an in-house manual drawing program in MATLAB (version 7.0; The MathWorks, Inc. Natick, Massachusetts, USA).

In the present study, the CT images were processed using 3 different radiomic feature extraction strategies. The first method of gray level histogram analysis (GLHA) was used to calculate six parameters (31): Maximum, minimum, mean, standard deviation, skewedness and kurtosis of the CT values inside the $3 \mathrm{D}$ VOI. These parameters reflect the basic statistical characteristics of pixels inside tumors. However, they do not take into consideration the spatial information between adjacent pixels.

The second method of spatial gray-level dependence matrices (SGLDM) was used to calculate 10 statistical measures to quantify the differences between two adjacent pixels along specific directions: Entropy, angular second moment, contrast, homogeneity, sum-mean, variance, correlation maximum probability, inverse difference moment and cluster tendency. These parameters have been defined by previous studies $(32,33)$. They are calculated from $2 \mathrm{D}$ co-occurrence matrices, whose function $\operatorname{Md}(\mathrm{i}, \mathrm{j})$ denotes the number of pairs of adjacent pixels with gray level $i$ and with gray level $\mathrm{j}$ along the dth direction. As CT images are too thick for detailed analysis, only adjacent pixels within the same transverse plane were considered. In the present study, four directions along $0^{\circ}, 45^{\circ}, 90^{\circ}$ and $135^{\circ}$ were used.

The third method of neighborhood gray-tone difference matrices (NGTDM) calculated five statistical parameters: Coarseness, contrast, business, complexity and strength. They have also been defined previously $(34,35)$. This method differs from SGLDM, which quantified the associations between two adjacent pixels along given axes. Instead, this method describes how the gray level of a pixel differs from that of all its neighbors. The parameters were calculated on the basis of a neighborhood gray-level difference matrix M(i). The elements of this matrix consist of the differences between a pixel with gray level $\mathrm{i}$ and all of its neighbors. As in SGLDM, a pixel's neighbors are only defined along the same transverse plane. Table I presents the radiomic features of these parameters, including category, label, feature name and description. Fig. 2 presents a diagram of the method adopted in the present study.

Statistical analysis. All statistical analyses were performed using SPSS version 19.0 (IBM Corp., Armonk, NY, USA). Student's t-tests were used for the comparison of two groups. 
Table I. Information of the 21 radiomic features.

Parameter category/label Feature name Description

Gray level histogram analysis

G1
G2
G3
G4
G5
G6

Spatial gray-level dependence matrix

S1
S2
S3
S4
S5
S6
S7
S8
S9
S10

Neighborhood gray-tone difference matrix

N1
N2
N3
N4
N5

Maximum of CT value

Minimum of $\mathrm{CT}$ value

Mean

Standard deviation

Skewedness

Kurtosis

\section{Entropy}

Angular second moment

Contrast

Homogeneity

Sum-mean

Variance

Correlation

Maximum probability

Inverse difference moment

Cluster tendency

\section{Coarseness}

Contrast

Frequency of involvement

Complexity

Intensity level
Six statistics calculated on gray-level distribution (histogram) without considering spatial associations of voxels

Ten parameters calculated from co-occurrence matrix, characterizing variations of gray levels for a pair of consecutive voxels by considering spatial associations

\section{CT, computed tomography.}

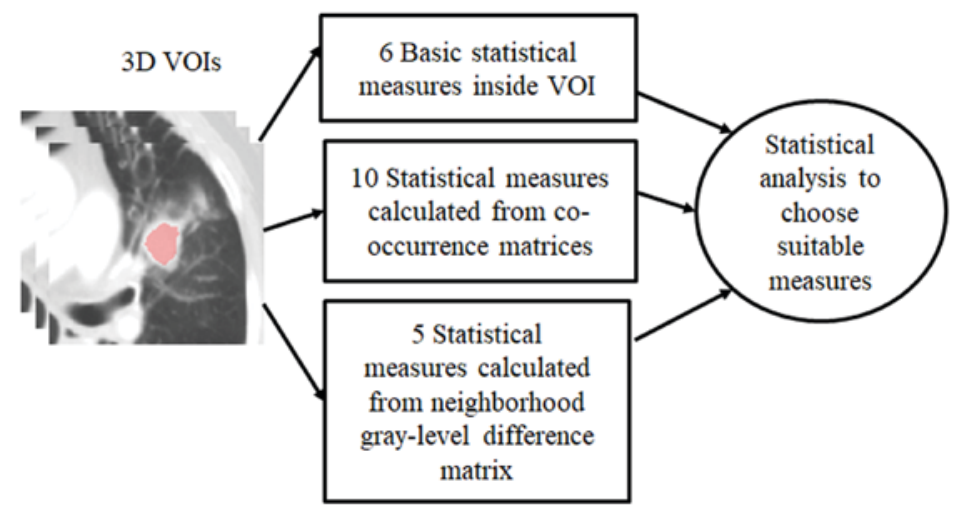

Figure 2. Flowchart of the radiomics feature extraction process. VOI, volume of interest; 3D, 3-dimensional.

Mann-Whitney U test was used to analyze the differences between each of the 21 computer-extracted features between the responsive and non-responsive two groups. Association between responsive and non-responsive groups and clinicopathological data from patients with SCLC was analyzed by Chi-square test. Binary logistic regression analysis was performed to assess the influence of the 21 computer-extracted indexes on the treatment efficacy. Receiver operating characteristic (ROC) curves were generated to determine the optimal cut-off threshold values.
The area under the curve (AUC) (36) was used to evaluate the accuracy of the radiomics signature and the model in predicting the efficacy of chemotherapy. $\mathrm{P} \leq 0.05$ was considered to indicate a statistically significant difference.

\section{Results}

Clinicopathological characteristics. The median age of the patients was 59 years (range, 22-83 years). Patients 
Table II. Association between clinicopathological parameters and patient outcome for patients with SCLC.

\begin{tabular}{|c|c|c|c|c|}
\hline Parameter & Total $(\mathrm{n}=92)$ & Response group $(\mathrm{n}=70)$ & No response group $(n=22)$ & P-value \\
\hline Age (years) & & & & 0.830 \\
\hline$<60$ & $52(56.5)$ & $40(57.1)$ & $12(54.5)$ & \\
\hline$\geq 60$ & $40(43.5)$ & $30(42.9)$ & $10(45.5)$ & \\
\hline Sex & & & & 0.546 \\
\hline Male & $74(80.4)$ & $55(78.6)$ & $19(86.4)$ & \\
\hline Female & $18(19.6)$ & $15(21.4)$ & $3(13.6)$ & \\
\hline Tumor extent & & & & 0.845 \\
\hline Limited & $36(39.1)$ & $27(38.6)$ & $9(40.9)$ & \\
\hline Extensive & $56(60.9)$ & $43(61.4)$ & $13(59.1)$ & \\
\hline Location & & & & 0.206 \\
\hline Central & $76(82.6)$ & $60(85.7)$ & $16(72.7)$ & \\
\hline Peripheral & $16(17.4)$ & $10(14.3)$ & $6(27.3)$ & \\
\hline T-stage & & & & 0.485 \\
\hline 0 & $18(19.6)$ & $13(18.6)$ & $5(22.7)$ & \\
\hline 1 & $26(28.3)$ & $18(25.7)$ & $8(36.4)$ & \\
\hline 2 & $30(32.6)$ & $23(32.9)$ & $7(31.8)$ & \\
\hline 3 & $18(19.6)$ & $16(22.9)$ & $2(9.1)$ & \\
\hline $\mathrm{N}$-stage & & & & 0.338 \\
\hline 0 & $5(5.4)$ & $4(5.7)$ & $1(4.5)$ & \\
\hline 1 & $11(12.0)$ & $6(8.6)$ & $5(22.7)$ & \\
\hline 2 & $39(42.4)$ & $30(42.9)$ & $9(40.9)$ & \\
\hline 3 & $37(40.2)$ & $30(42.9)$ & $7(31.8)$ & \\
\hline M-stage & & & & 0.206 \\
\hline 0 & $52(56.5)$ & $37(52.9)$ & $15(68.2)$ & \\
\hline 1 & $40(43.5)$ & $33(47.1)$ & $7(31.8)$ & \\
\hline Smoking & & & & 0.050 \\
\hline No & $28(30.4)$ & $25(35.7)$ & $3(13.6)$ & \\
\hline Yes & $64(69.6)$ & $45(64.3)$ & $19(86.4)$ & \\
\hline Smoking index & & & & 0.100 \\
\hline$<400$ & $39(42.4)$ & $33(47.1)$ & $6(27.3)$ & \\
\hline$\geq 400$ & $53(57.6)$ & $37(52.9)$ & $16(72.7)$ & \\
\hline Smoking time (years) & & & & 0.378 \\
\hline$\leq 10$ & $30(32.6)$ & $27(38.6)$ & $3(13.6)$ & \\
\hline $11-20$ & $15(16.3)$ & $10(14.3)$ & $5(22.7)$ & \\
\hline $21-30$ & $22(23.9)$ & $16(22.9)$ & $6(27.3)$ & \\
\hline$\geq 30$ & $25(27.2)$ & $17(24.3)$ & $8(36.4)$ & \\
\hline ProGRP (ng/ml) & & & & 0.078 \\
\hline$<54.8$ & $71(77.2)$ & $51(72.9)$ & $20(90.9)$ & \\
\hline$\geq 54.8$ & $21(22.8)$ & $19(27.1)$ & $2(9.1)$ & \\
\hline NSE (ng/ml) & & & & 0.275 \\
\hline$<3.4$ & $19(20.7)$ & $13(18.6)$ & $6(27.3)$ & \\
\hline$\geq 3.4$ & $73(79.3)$ & $57(81.4)$ & $16(72.7)$ & \\
\hline CEA (ng/ml) & & & & 0.337 \\
\hline$<17$ & $50(54.3)$ & $40(57.1)$ & $10(45.5)$ & \\
\hline$\geq 17$ & $42(45.7)$ & $30(42.9)$ & $12(54.5)$ & \\
\hline Cyfra21-1 (ng/ml) & & & & 0.512 \\
\hline$<3.3$ & $53(57.6)$ & $39(55.7)$ & $14(63.6)$ & \\
\hline$\geq 3.3$ & $39(42.4)$ & $31(44.3)$ & $8(36.4)$ & \\
\hline
\end{tabular}

Values are expressed as n (\%). T-stage, tumor stage; N-stage, node stage; M-stage, metastasis stage; ProGRP, precursors of gastrin release peptide; NSE, neuronspecific enolase; CEA, carcinoembryonic antigen; Cyfra21-1, cytokeratin 19 fragment. 
Table III. Univariate analysis of the 21 radiomic features associated with the chemotherapeutic effect introduced in Table II.

\begin{tabular}{lccc}
\hline Radiomic feature & Mean \pm SD & Z & P-value \\
\hline G1 & $119.750 \pm 12.976$ & -2.325 & 0.020 \\
G2 & $1.110 \pm 4.835$ & -0.175 & 0.861 \\
G3 & $63.080 \pm 4.222$ & -1.895 & 0.058 \\
G4 & $9.600 \pm 4.677$ & -0.989 & 0.323 \\
G5 & $-0.700 \pm 1.103$ & -1.071 & 0.284 \\
G6 & $10.760 \pm 6.485$ & -1.510 & 0.131 \\
S1 & $6.240 \pm 0.407$ & -0.622 & 0.534 \\
S2 & $0.004 \pm 0.063$ & -0.201 & 0.840 \\
S3 & $66.050 \pm 47.271$ & -1.565 & 0.118 \\
S4 & $0.300 \pm 0.0357$ & -1.245 & 0.213 \\
S5 & $64.370 \pm 4.199$ & -1.922 & 0.055 \\
S6 & $102.410 \pm 168.990$ & -1.044 & 0.297 \\
S7 & $0.570 \pm 0.111$ & -0.211 & 0.833 \\
S8 & $0.015 \pm 0.030$ & -0.842 & 0.400 \\
S9 & $0.200 \pm 0.037$ & -2.087 & 0.037 \\
S10 & $343.590 \pm 640.074$ & -0.86 & 0.390 \\
N1 & $0.012 \pm 0.005$ & -0.32 & 0.749 \\
N2 & $0.086 \pm 0.145$ & -1.556 & 0.120 \\
N3 & $0.530 \pm 0.530$ & -0.137 & 0.891 \\
N4 & $1.740 \pm 3.782$ & -0.824 & 0.410 \\
N5 & $4.660 \pm 7.240$ & -0.165 & 0.869 \\
& & &
\end{tabular}

Values are expressed as the mean \pm standard deviation $(n=92)$.

aged $\geq 60$ years accounted for $43.5 \%$ of the study population and the majority of the patients were male $(n=74$; $80.4 \%$ ). Smokers accounted for $69.6 \%$ of the population and 40 patients $(43.5 \%)$ presented with distant metastasis (Table II).

Association of clinicopathological factors with treatment efficacy. The smoking status in the response group was significantly associated with treatment efficacy compared with the no response group $(\mathrm{P}=0.05)$. However, there was no statistical significance in the smoking time $(\leq 10,11-20,21-30$ and $\geq 30$ years, respectively; $38.6,14.3,22.8$ and 24.3 vs. 13.6, 22.7, 27.3 and $36.4 \%)$ and smoking index $(<400$ and $\geq 400$, respectively; 47.1 and 52.9 vs. 27.3 and $72.7 \%$ ) between the response and no response groups ( $\mathrm{P}>0.05$; Table II). This may be due to the small size of the cohort, which limits the statistical power of the data.

Association of the 21 radiomics characteristics with treatment efficacy. Univariate analysis revealed that, among the 21 radiological features analyzed, two features exhibited significant differences between the response and no response groups. The GLHA category had a maximum CT value, which was significantly different between the two groups $(\mathrm{P}=0.020)$. The inverse difference moment was observed in the SGLDM category, which was also significantly different between the two groups $(\mathrm{P}=0.037$; Table III).
Table IV. Prediction model of 21 radiomics features.

\begin{tabular}{lrcr}
\hline $\begin{array}{l}\text { Radiomic } \\
\text { feature }\end{array}$ & $\beta$ & Odds ratio $(95 \% \mathrm{CI})$ & P-value \\
\hline G2 & 0.176 & $1.193(0.940-1.514)$ & 0.147 \\
G4 & 2.088 & $8.069(2.077-31.340)$ & 0.003 \\
S3 & -0.139 & $0.870(0.790-0.959)$ & 0.005 \\
S5 & 0.193 & $1.213(0.962-1.531)$ & 0.103 \\
S7 & -14.174 & $<0.001(0-0.248)$ & 0.030 \\
N2 & -59.356 & $<0.001(0-<0.001)$ & 0.002 \\
N3 & 1.661 & $5.266(0.672-41.244)$ & 0.114 \\
N4 & 1.605 & $4.979(1.790-13.847)$ & 0.002 \\
\hline
\end{tabular}

The radiomics model shows the results of 21 radiomics features $(n=92) . \beta$, regression coefficient; CT, computed tomography; CI, confidence interval.

Table V. Multivariate analysis in the backward logistic regression model of clinicopathological parameters.

\begin{tabular}{lccc}
\hline Parameter & $\beta$ & Odds ratio (95\% CI) & P-value \\
\hline $\begin{array}{l}\text { Gender (female) } \\
\text { Tumor extent }\end{array}$ & -0.764 & $0.466(0.056-3.869)$ & 0.479 \\
(extensive) & & $0.474(0.113-1.996)$ & 0.309 \\
Tumor location & -0.587 & $0.556(0.124-2.482)$ & 0.442 \\
(peripheral) & & & \\
T-stage (T0) & & & 0.323 \\
T1 & -1.173 & $0.309(0.038-2.547)$ & 0.275 \\
T2 & -1.903 & $0.149(0.020-1.111)$ & 0.063 \\
T3 & -1.296 & $0.274(0.043-1.759)$ & 0.172 \\
M-stage (M0) & 0.836 & $2.306(0.599-8.877)$ & 0.224 \\
Smoking (yes) & -2.024 & $0.132(0.020-0.894)$ & 0.038 \\
ProGRP ( $\geq 54.8)$ & 0.789 & $2.202(0.385-12.600)$ & 0.375 \\
NSE ( $\geq 3.4)$ & 0.651 & $1.917(0.500-7.345)$ & 0.342 \\
\hline
\end{tabular}

The clinical model provides the results of multiple factors of clinical risk factors for small cell lung cancer $(n=92)$. $\beta$, regression coefficient; T-stage, tumor-stage (T0); M-stage, metastasis stage (M0); ProGRP, precursors of gastrin release peptide; NSE, neuronspecific enolase; CI, confidence interval.

\section{Establishment of prediction models of the response to SCLC chemotherapy}

Prediction results of the radiomic features model. The radiomics signature model took into account 21 radiomics features. In the binary logistic regression analysis, backward logistic regression was adopted $\left(\mathrm{R}^{2}=0.358\right)$. Analysis of the joint radiomics parameters revealed that the following 5 radiomics features exhibited significant differences: i) characteristics of the GLHA category [standard deviation, $\mathrm{P}=0.003$; odds ratio, 8.069; 95\% confidence interval (CI), 2.077-31.340]; ii) characteristics of the SGLDM category (contrast, $\mathrm{P}=0.005$; odds ratio, $0.870 ; 95 \% \mathrm{CI}, 0.790-0.959$ and correlation, 


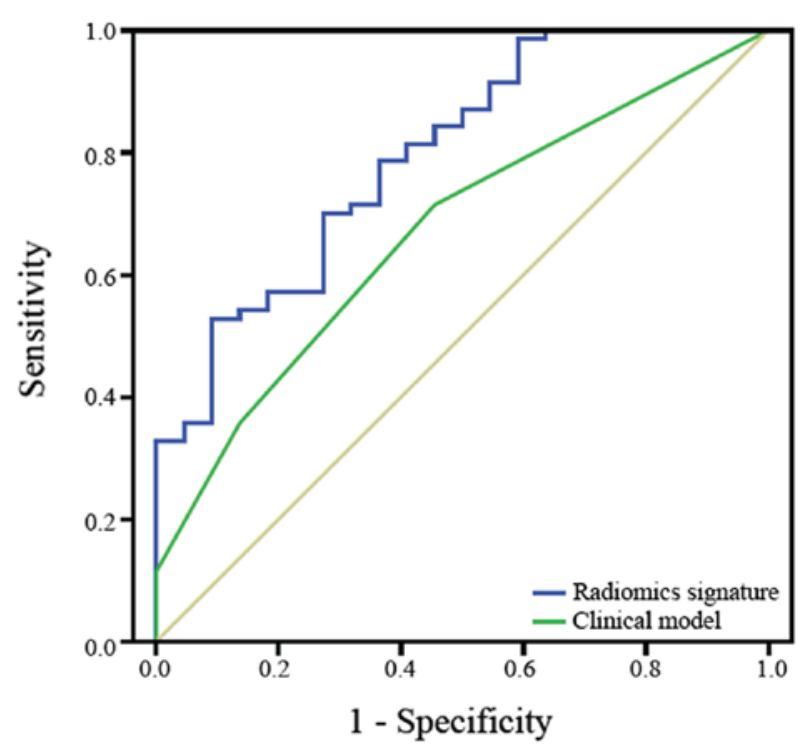

Figure 3. Receiver operating characteristic curves of the radiomics signature (blue line) and clinical model (green line).

$\mathrm{P}=0.030$; odds ratio, $<0.001 ; 95 \%$ CI $0-0.248)$; iii) characteristics of the NGTDM category (contrast, $\mathrm{P}=0.002$; odds ratio, $<0.001,95 \%$ CI, 0-0.001); and complexity (N4, P=0.002; odds ratio, $4.979 ; 95 \% \mathrm{CI}, 1.790-13.847$; Table IV).

Prediction results of the clinicopathological parameter model. The clinicopathological parameter model took into account conventional clinical risk factors (age $\geq 60$ years, male sex, extensive tumor, central tumor, T1-3, N1-3, M1, smoking, smoking time, smoking index $\geq 400$, ProGRP $\geq 54.8 \mathrm{ng} / \mathrm{ml}$, $\mathrm{NSE} \geq 3.4 \mathrm{ng} / \mathrm{ml}, \mathrm{CEA} \geq 17 \mathrm{ng} / \mathrm{ml}$ and Cyfra $21-1 \geq 3.3 \mathrm{ng} / \mathrm{ml}$ ). For the binary logistic regression analysis, a backward logistic regression was adopted $\left(\mathrm{R}^{2}=0.121\right)$. The results revealed that only smoking had a significant impact on the response to chemotherapy $(\mathrm{P}=0.038$; 95\% CI, 0.020-0.894; Table V).

Predictive performance of the two models. The ROC curves of the radiomics signature model and clinicopathological parameter model were calculated using the significant variables in the logistic regression and are presented in Fig. 3. The AUC of the radiomics signature was 0.797 (95\% CI, 0.692-0.901). The AUC of the clinicopathological parameter model was 0.670 (95\% CI, 0.547-0.793). Regarding the prediction of the accuracy of the efficacy of chemotherapy in patients with SCLC, the radiomics signature model was superior to the clinicopathological parameter model.

\section{Discussion}

In the subgroup of current and former smokers, the lung cancer-associated mortality rate is highest at $20 \%$. Smoking is an important independent risk factor for SCLC and $\geq 90 \%$ of patients with SCLC are smokers $(1,37)$. In the present study, a significant difference in response to treatment between the smoking and non-smoking groups was identified. However, the smoking time and smoking index exhibited a similar trend but there was no significant difference observed. This may be due to the small size of the cohort, which limits the statistical power of the data. The results of the present study demonstrated that smoking was associated with the efficacy of chemotherapy in patients with SCLC. Thus, smoking cessation should be encouraged by clinicians.

At present, the tumor response is assessed by determining changes in tumor size (RECIST 1.1 is based on the diameter of the lesion). However, the tumor size does not truly reflect the morphological, functional and metabolic changes following tumor treatment, as necrosis, hemorrhage and cavitation frequently occur following chemotherapy (38). The present study, statistical analysis of primary tumor and radiomic texture characteristics may be used to predict the efficacy of chemotherapy in patients with SCLC. Such analyses constitute a powerful upgrade of the morphological assessment system, as indicated in a study on the pathological responses of lung cancer patients to chemotherapy (39).

Radiomics provides a non-invasive, rapid, cost-effective and reproducible method for detailed and comprehensive characterization of the tumor phenotypic information (shape, intensity, texture and wavelets) $(17-19,36,40,41)$. It may reduce the requirement for biopsy and it appears to offer a nearly limitless supply of imaging information that may potentially aid in the prediction of the response to chemotherapy as early as possible and, in turn, reduce unnecessary chemotherapy (42). Recently, radiomics has made a series of advances in tumor prediction. A radiomics signature has been demonstrated to be a biomarker for distant metastasis of lung adenocarcinoma $(\mathrm{AUC}=0.61)(25)$. It is able to predict histopathological features of lung carcinoma $(22,23,43,44)$, the recurrence of hepatocellular carcinoma $(\mathrm{AUC}=0.817)(45)$ and pre-operative prediction of lymph node metastasis in colorectal cancer $(\mathrm{AUC}=0.736)$ (46). Radiomics studies of lung cancer have mostly focused on non-SCLC $(20,22-26,43)$; however, to the best of our knowledge, no previous study has investigated radiomics approaches in SCLC.

Radiomics features may be divided into four categories: Morphology, statistics, region and model. The statistical features of these categories may be classified as single-point statistics (histogram) or higher-order statistics (texture) (47). In the present study, 21 quantitative parameters were extracted from the statistical radiology characteristics. GLHA features constitute single-point statistics, which include 6 statistics (maximum of CT value, minimum of CT value, mean, standard deviation, skewedness and kurtosis) calculated from gray-level distributions (histogram) of voxels without considering spatial associations. SGLDM parameters include 10 statistics calculated from a co-occurrence matrix, characterizing variations of gray levels for a pair of consecutive voxels by considering spatial associations. NGTDM parameters use the intensity values of a neighborhood instead of one pixel to characterize differences in gray levels between a voxel and all its neighbors. SGLDM and NGTDM constitute higher-order statistics. They may be used to obtain information regarding the spatial association between pixels and thus reflect the textural characteristics of tumors. Ultimately, the five characteristics that exhibited significant differences between the effective and ineffective groups were included in the present model; the first- and higher-order statistics were included. Of note, the radiological features prediction model based on these features 
$(\mathrm{AUC}=0.797)$ had a higher predictive accuracy than the model of clinicopathological characteristics $(\mathrm{AUC}=0.670)$, indicating that radiomics features provide more information regarding heterogeneity within a tumor, and that the radiomics signature may successfully stratify chemotherapy patients into high- and low-risk groups. This would allow for the targeted and continued treatment of patients expected to exhibit a poor response to first-line chemotherapy, and may improve long-term patient survival.

In conclusion, the results of the present study revealed that a radiomics signature may be an independent predictor of the efficacy of chemotherapy in SCLC patients. It represents a novel biomarker that may be used for quantitative analysis in radiology and this information may help clinicians to better evaluate patients and select optimal treatment strategies.

\section{Acknowledgements}

Not applicable.

\section{Funding}

The present study was supported by grants from the Taishan Scholars Project, National Natural Science Foundation Research (grant nos. 81371548, 81571672 and 61603218), and the Shandong Provincial Natural Science Foundation (grant no. ZR2014HQ054).

\section{Availability of data and materials}

The datasets used and/or analyzed during the current study are available from the corresponding author on reasonable request.

\section{Authors' contributions}

$\mathrm{XW}, \mathrm{XQ}$ and HW contributed to the conception/design of the study; HW, FY and ZL acquired the data; HW, FY, ZL, SS, FX, HL, PL and XW analyzed the data; HW, QL and XQ prepared the manuscript. QL also made substantial contributions to acquisition, analysis and interpretation of data All authors critically revised and approved the final version of the manuscript.

\section{Ethics approval and consent to participate}

The current study was performed in accordance with the Declaration of Helsinki. The present study was approved by the Ethics Committee of Shandong Cancer Hospital Affiliated to Shandong University (Jinan, China) and written informed consent was obtained from each participant prior to the study.

\section{Patient consent for publication}

Not applicable.

\section{Competing interests}

The authors declare that they have no competing interests.

\section{References}

1. Siegel RL, Miller KD and Jemal A: Cancer Statistics, 2017. CA Cancer J Clin 67: 7-30, 2017.

2. Chen W, Zheng R, Baade PD, Zhang S, Zeng H, Bray F, Jemal A, $\mathrm{Yu}$ XQ and He J: Cancer statistics in China, 2015. CA Cancer J Clin 66: 115-132, 2016.

3. van Meerbeeck JP, Fennell DA and De Ruysscher DK: Small-cell lung cancer. Lancet 378: 1741-1755, 2011.

4. Alvarado-Luna G and Morales-Espinosa D: Treatment for small cell lung cancer, where are we now?-a review. Transl Lung Cancer Res 5: 26-38, 2016.

5. Bunn PA Jr, Minna JD, Augustyn A, Gazdar AF, Ouadah Y, Krasnow MA, Berns A, Brambilla E, Rekhtman N, Massion PP, et al: Small cell lung cancer: Can recent advances in biology and molecular biology be translated into improved outcomes? J Thorac Oncol 11: 453-474, 2016.

6. Polański R, Hodgkinson CL, Fusi A, Nonaka D, Priest L, Kelly P, Trapani F, Bishop PW, White A, Critchlow SE, et al: Activity of the monocarboxylate transporter 1 inhibitor AZD3965 in small cell lung cancer. Clin Cancer Res 20: 926-937, 2014.

7. Sen T, Tong P, Stewart CA, Cristea S, Valliani A, Shames DS, Redwood AB, Fan YH, Li L, Glisson BS, et al: CHK1 inhibition in small-cell lung cancer produces single-agent activity in biomarker-defined disease subsets and combination activity with cisplatin or olaparib. Cancer Res 77: 3870-3884, 2017.

8. Ross JS, Wang K, Elkadi OR, Tarasen A, Foulke L, Sheehan CE, Otto GA, Palmer G, Yelensky R, Lipson D, et al: Next-generation sequencing reveals frequent consistent genomic alterations in small cell undifferentiated lung cancer. J Clin Pathol 67: 772-776, 2014.

9. Qiu YF, Liu ZG, Yang WJ, Zhao Y, Tang J, Tang WZ, Jin Y, $\mathrm{Li} F$, Zhong R and Wang H: Research progress in the treatment of small cell lung cancer. J Cancer 8: 29-38, 2017.

10. Lu HY, Wang XJ and Mao WM: Targeted therapies in small cell lung cancer. Oncol Lett 5: 3-11, 2013.

11. Hiltermann TJ, Pore MM, van den Berg A, Timens W, Boezen HM, Liesker JJ, Schouwink JH, Wijnands WJ, Kerner GS, Kruyt FA, et al: Circulating tumor cells in small-cell lung cancer: A predictive and prognostic factor. Ann Oncol 23: 2937-2942, 2012.

12. Hou JM, Krebs MG, Lancashire L, Sloane R, Backen A, Swain RK, Priest LJ, Greystoke A, Zhou C, Morris K, et al: Clinical significance and molecular characteristics of circulating tumor cells and circulating tumor microemboli in patients with small-cell lung cancer. J Clin Oncol 30: 525-532, 2012.

13. Zhu LR, Li J, Chen P, Jiang Q and Tang XP: Clinical significance of plasma fibrinogen and D-dimer in predicting the chemotherapy efficacy and prognosis for small cell lung cancer patients. Clin Transl Oncol 18: 178-188, 2016.

14. Yang J, Jiao S, Kang J, Li R and Zhang G: Application of serum NY-ESO-1 antibody assay for early SCLC diagnosis. Int J Clin Exp Pathol 8: 14959-14964, 2015.

15. Buil-Bruna N, López-Picazo JM, Moreno-Jiménez M, Martin-Algarra S, Ribba B and Trocóniz IF: A population pharmacodynamic model for lactate dehydrogenase and neuron specific enolase to predict tumor progression in small cell lung cancer patients. AAPS J 16: 609-619, 2014.

16. Gillies RJ, Kinahan PE and Hricak H: Radiomics: Images are more than pictures, they are data. Radiology 278: 563-577, 2016.

17. Lambin P, Rios-Velazquez E, Leijenaar R, Carvalho S, van Stiphout RG, Granton P, Zegers CM, Gillies R, Boellard R, Dekker A and Aerts HJ: Radiomics: Extracting more information from medical images using advanced feature analysis. Eur J Cancer 48: 441-446, 2012.

18. Chen B, Zhang R, Gan Y, Yang L and Li W: Development and clinical application of radiomics in lung cancer. Radiat Oncol 12: $154,2017$.

19. Aerts HJ, Velazquez ER, Leijenaar RT, Parmar C, Grossmann P, Carvalho S, Bussink J, Monshouwer R, Haibe-Kains B, Rietveld $\mathrm{D}$, et al: Decoding tumour phenotype by noninvasive imaging using a quantitative radiomics approach. Nat Commun 5: 4006, 2014.

20. Fave X, Zhang L, Yang J, Mackin D, Balter P, Gomez D, Followill D, Jones AK, Stingo F, Liao Z, et al: Delta-radiomics features for the prediction of patient outcomes in non-small cell lung cancer. Sci Rep 7: 588, 2017.

21. Wang H, Guo XH, Jia ZW, Li HK, Liang ZG, Li KC and He Q: Multilevel binomial logistic prediction model for malignant pulmonary nodules based on texture features of CT image. Eur J Radiol 74: 124-129, 2010. 
22. Ferreira Junior JR, Koenigkam-Santos M, Cipriano FEG, Fabro AT and Azevedo-Marques PM: Radiomics-based features for pattern recognition of lung cancer histopathology and metastases. Comput Methods Programs Biomed 159: 23-30, 2018.

23. Wu W, Parmar C, Grossmann P, Quackenbush J, Lambin P, Bussink J, Mak R and Aerts HJ: Exploratory study to identify radiomics classifiers for lung cancer histology. Front Oncol 6: 71, 2016.

24. Ganeshan B, Abaleke S, Young RC, Chatwin CR and Miles KA Texture analysis of non-small cell lung cancer on unenhanced computed tomography: Initial evidence for a relationship with tumour glucose metabolism and stage. Cancer Imaging 10: 137-143, 2010.

25. CorollerTP,Grossmann P,Hou Y,Rios Velazquez E, Leijenaar RT, Hermann G, Lambin P, Haibe-Kains B, Mak RH and Aerts HJ: CT-based radiomic signature predicts distant metastasis in lung adenocarcinoma. Radiother Oncol 114: 345-350, 2015.

26. Fried DV, Tucker SL, Zhou S, Liao Z, Mawlawi O, Ibbott G and Court LE: Prognostic value and reproducibility of pretreatment $\mathrm{CT}$ texture features in stage III non-small cell lung cancer. Int J Radiat Oncol Biol Phys 90: 834-842, 2014.

27. Gevaert O, Xu J, Hoang CD, Leung AN, Xu Y, Quon A, Rubin DL, Napel S and Plevritis SK: Non-small cell lung cancer: Identifying prognostic imaging biomarkers by leveraging public gene expression microarray data-methods and preliminary results. Radiology 264: 387-396, 2012.

28. Eisenhauer EA, Therasse P, Bogaerts J, Schwartz LH, Sargent D, Ford R, Dancey J, Arbuck S, Gwyther S, Mooney M, et al: New response evaluation criteria in solid tumours: Revised RECIST guideline (version 1.1). Eur J Cancer 45: 228-247, 2009.

29. Ohno Y, Fujisawa Y, Koyama H, Kishida Y, Seki S, Sugihara N and Yoshikawa T: Dynamic contrast-enhanced perfusion area-detector CT assessed with various mathematical models: Its capability for therapeutic outcome prediction for non-small cell lung cancer patients with chemoradiotherapy as compared with that of FDG-PET/CT. Eur J Radiol 86: 83-91, 2017.

30. Zelen M: Keynote address on biostatistics and data retrieval Cancer Chemother Rep 3 4: 31-42, 1973.

31. Huang X, Cheng Z, Huang Y, Liang C, He L, Ma Z, Chen X, Wu X, Li Y, Liang C and Liu Z: CT-based radiomics signature to discriminate high-grade from low-grade colorectal adenocarcinoma. Acad Radiol 25: 1285-1297, 2018.

32. Haralick RM, Shanmugam K and Dinstein I: Textural features for image classification. IEEE Trans Syst Man Cybern 3: 610-621, 1973.

33. Kurani AS, Xu DH, Furst J and Raicu DS: Co-occurrence matrices for volumetric data. In: Proceedings of the 7th IASTED International Conference on Computer Graphics and Imaging. CGIM, Kauai, Hawaii, pp447-452, 2004.

34. Amadasun M and King R: Textural features corresponding to textural properties. IEEE Trans Systems Man Cybern 19: 1264-1274, 1989.

35. Xu R, Kido S, Suga K, Hirano Y, Tachibana R, Muramatsu K, Chagawa K and Tanaka S: Texture analysis on (18)F-FDG PET/CT images to differentiate malignant and benign bone and soft-tissue lesions. Ann Nucl Med 28: 926-935, 2014.
36. van Griethuysen JJM, Fedorov A, Parmar C, Hosny A, Aucoin N, Narayan V, Beets-Tan RGH, Fillion-Robin JC, Pieper S and Aerts HJWL: Computational radiomics system to decode the radiographic phenotype. Cancer Res 77: e104-e107, 2017.

37. Pesch B, Kendzia B, Gustavsson P, Jöckel KH, Johnen G, Pohlabeln H, Olsson A, Ahrens W, Gross IM, Brüske I, et al: Cigarette smoking and lung cancer-relative risk estimates for the major histological types from a pooled analysis of case-control studies. Int J Cancer 131: 1210-1219, 2012.

38. Kang H, Lee HY, Lee KS and Kim JH: Imaging-based tumor treatment response evaluation: Review of conventional, new, and emerging concepts. Korean J Radiol 13: 371-390, 2012.

39. Chong Y, Kim JH, Lee HY, Ahn YC, Lee KS, Ahn MJ, Kim J, Shim YM, Han J and Choi YL: Quantitative CT variables enabling response prediction in neoadjuvant therapy with EGFR-TKIs: Are they different from those in neoadjuvant concurrent chemoradiotherapy? PLoS One 9: e88598, 2014.

40. Zhong Y, Yuan M, Zhang T, Zhang YD, Li H and Yu TF: Radiomics approach to prediction of occult mediastinal lymph node metastasis of lung adenocarcinoma. AJR Am J Roentgenol 211: 109-113, 2018.

41. Bogowicz M, Riesterer O, Ikenberg K, Stieb S, Moch H, Studer G, Guckenberger $\mathrm{M}$ and Tanadini-Lang S: Computed tomography radiomics predicts HPV status and local tumor control after definitive radiochemotherapy in head and neck squamous cell carcinoma. Int J Radiat Oncol Biol Phys 99: 921-928, 2017.

42. Avanzo M, Stancanello J and El Naqa I: Beyond imaging: The promise of radiomics. Phys Med 38: 122-139, 2017.

43. Ha S, Choi H, Cheon GJ, Kang KW, Chung JK, Kim EE and Lee DS: Autoclustering of non-small cell lung carcinoma subtypes on (18)F-FDG PET using texture analysis: A preliminary result. Nucl Med Mol Imaging 48: 278-286, 2014.

44. Patil R, Mahadevaiah G and Dekker A: An approach toward automatic classification of tumor histopathology of non-small cell lung cancer based on radiomic features. Tomography 2: 374-377, 2016

45. Zhou Y, He L, Huang Y, Chen S, Wu P, Ye W, Liu Z and Liang C: CT-based radiomics signature: a potential biomarker for preoperative prediction of early recurrence in hepatocellular carcinoma. Abdom Radiol (NY) 42: 1695-1704, 2017

46. Huang YQ, Liang CH, He L, Tian J, Liang CS, Chen X, Ma ZL and Liu ZY: Development and validation of a radiomics nomogram for preoperative prediction of lymph node metastasis in colorectal cancer. J Clin Oncol 34: 2157-2164, 2016.

47. Lee G, Lee HY, Park H, Schiebler ML, van Beek EJR, Ohno Y, Seo JB and Leung A: Radiomics and its emerging role in lung cancer research, imaging biomarkers and clinical management: State of the art. Eur J Radiol 86: 297-307, 2017.

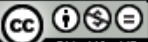

This work is licensed under a Creative Commons Attribution-NonCommercial-NoDerivatives 4.0 International (CC BY-NC-ND 4.0) License. 